\title{
Predictive and protective role of high-density lipoprotein cholesterol in acute myocardial infarction
}

\author{
Jin Sup Park ${ }^{1,2}$, Kwang Soo Cha ${ }^{1,2}$, Hye Won Lee ${ }^{1}$, Jun-Hyok Oh ${ }^{1}$, Jung Hyun Choi ${ }^{1}$, \\ Han Cheol Lee ${ }^{1}$, Taek Jong Hong ${ }^{1}$, Myung Ho Jeong ${ }^{3}$, Shung Chull Chae ${ }^{4}$, Young Jo Kim ${ }^{5}$; \\ the Korean Acute Myocardial Infarction Registry Investigators

\begin{abstract}
${ }^{1}$ Department of Cardiology, Pusan National University Hospital, Busan, South Korea
${ }^{2}$ Medical Research Institute, Pusan National University Hospital, Busan, South Korea

${ }^{5}$ Department of Cardiology, Yeungnam University Hospital, Daegu, South Korea
\end{abstract} \\ ${ }^{3}$ Department of Cardiology, Chonnam National University Hospital, Gwangju, South Korea \\ ${ }^{4}$ Department of Cardiology, Kyungpook National University Hospital, Daegu, South Korea
}

\begin{abstract}
Background: It is unclear whether high-density lipoprotein cholesterol (HDL-C) level predicts cardiovascular events and has a protective effect in patients with acute myocardial infarction (AMI) undergoing percutaneous coronary intervention (PCI) and statin treatment.

Methods: A total of 15,290 AMI patients receiving statins were selected from the Korean Myocardial Infarction Registry. Baseline HDL-C level was used to identify patients with low (group A), normal (group B), and high (group C) HDL-C levels according to the Adult Treatment Panel III criteria. Clinical outcomes were compared in propensity-adjusted and matched cohorts. The primary endpoint was a composite of cardiovascular death and recurrent myocardial infarction.

Results: At the median follow-up of 11.5 months, the primary endpoint occurred in 2.7\% (112/4098), $1.4 \%$ (54/3910), and 1.2\% (8/661) of patients in groups A, B, and C, respectively. In the propensity-adjusted cohort, low HDL-C level increased the risk of primary endpoint (hazard ratio [HR] 1.755, 95\% confidence interval [CI] 1.274-2.417, $p=0.001$ ), whereas high HDL-C level did not reduce this risk (HR 0.562, 95\% CI 0.275-1.146, $p=0.113$ ). In the propensity-matched cohort, low HDL-C level increased the risk of primary endpoint (HR 1.716, 95\% CI 1.210-2.434, $p=0.002)$, whereas high $H D L-C$ level reduced this risk (HR 0.449, 95\% CI 0.214-0.946, $p=0.035$ ).

Conclusions: In AMI patients treated with PCI and statins, low HDL-C level increases the risk of cardiovascular death and recurrent myocardial infarction, whereas high $H D L-C$ level likely reduces the risk of cardiovascular events, especially for ST-elevation myocardial infarction. (Cardiol J 2019; 26, 2: 176-185)
\end{abstract}

Key words: high-density lipoprotein cholesterol, acute myocardial infarction, cardiovascular events, statin

\section{Introduction}

Several randomized control trials have shown that persistent cardiovascular risk remains in spite of reducing the level of low-density lipoprotein cho- lesterol (LDL-C) with intensive statin therapy [1]. In contrast, an inverse relation between the level of high-density lipoprotein cholesterol (HDL-C) and the rate of cardiovascular events has been revealed in a number of studies [2, 3]. A high

Address for correspondence: Kwang Soo Cha, MD, PhD, Department of Cardiology and Medical Research Institute, Pusan National University Hospital, 1-10 Ami-dong Seo-gu, Busan 602-739, Republic of Korea, tel: 82-10-4569-5879, fax: 82-51-247-5875, e-mail: chakws1@hanmail.net

Received: 26.05.2016

Accepted: 11.08.2016 
HDL-C level is associated with cardioprotective and anti-inflammatory effects, and achieving it represents a potential therapeutic strategy to reduce cardiovascular risk [4].

Whether low HDL-C levels predict poor cardiovascular outcomes in acute myocardial infarction (AMI) patients has been a matter of controversy $[5,6]$. In this regard, vascular effects of HDL-C can be highly heterogeneous in various clinical conditions [7], and its anti-inflammatory function may be impaired in patients with coronary artery disease (CAD) [8]. It is therefore important to verify the protective role of high HDL-C levels in patients with AMI.

In the present study, the predictive and protective role of HDL-C in a cohort of Asian patients with AMI undergoing percutaneous coronary intervention (PCI) and intensive statin therapy were evaluated.

\section{Methods}

\section{Study population}

In this prospective, multicenter, observational registry-based study, the data of 31,149 patients with ST-elevation myocardial infarction (STEMI) and non-STEMI (NSTEMI) from 53 hospitals were retrieved from the Korean Acute Myocardial Infarction Registry (KAMIR) between 2006 and $2012[9,10]$. This was retrospective study based on the KAMIR registry. The 53 participating centers included high-volume university or community hospitals with facilities for PCI and on-site cardiac surgery. The study protocol conformed to the ethical guidelines of the 1975 Declaration of Helsinki as reflected by prior approval by the human research committee of each participating institution. Informed consent for use of data was obtained from each patient. Clinical and laboratory characteristics and outcomes were collected by trained study coordinators using a standardized case report form and protocol. Angiographic characteristics such as American College of Cardiology/American Heart Association (ACC/AHA) lesion type or thrombolysis in myocardial infarction (TIMI) flow grade were assessed by the surgeons. Clinical follow-up was performed at $1,2,6,12$, and 24 months after discharge from hospital.

The inclusion criteria for the present study were as follows: (1) age $\geq 18$ years; (2) diagnosis of STEMI or NSTEMI; (3) absence of clinical events during hospitalization; and (4) statins use at discharge. The exclusion criterion was missing information on serum HDL-C level.
Among the 31,149 patients registered in the above mentioned database, a total of 15,290 AMI patients treated with statins were eligible for this study. The patients were divided into three groups based on serum HDL-C levels at baseline according to the Adult Treatment Panel III (ATP III) guidelines (group A: 7,308 patients with low HDL-C, group B: 6,827 patients with normal HDL-C, group C: 1,155 patients with high HDL-C) (Fig. 1). The median follow-up period was 347 days (interquartile range: 59-403 days). Follow-up information was obtained in 14,830 patients $(97 \%$, excluding 460 patients who died during hospitalization) among the initial 15,290 patients.

\section{Definitions and outcomes}

Blood samples were collected at admission, except for the samples used for obtaining lipid profiles, which were collected after overnight fasting.

According to the ATP III guidelines [11], a low HDL-C level (group A) was defined as a concentration of HDL-C below $40 \mathrm{mg} / \mathrm{dL}$ for men and $50 \mathrm{mg} / \mathrm{dL}$ for women. A high HDL-C level (group C) was defined as a concentration of HDL-C over $60 \mathrm{mg} / \mathrm{dL}$. A normal HDL-C level (group B) was defined as an HDL-C concentration between the limits of the low and high HDL-C groups.

The primary outcome was the composite of cardiovascular death and recurrent MI during the follow-up period. Secondary outcomes included cardiovascular death, all-cause death, recurrent MI, any revascularization, and major adverse cardiac events (MACE), which included all-cause death, recurrent $\mathrm{MI}$, and any revascularization during the follow-up. All events were identified by the patient's physician and confirmed by the principal investigator of each hospital.

\section{Statistical analyses}

Continuous variables were compared using Student's t-test or the Wilcoxon rank-sum test, and categorical variables were compared using the $\chi^{2}$ test or Fisher's exact test, as appropriate. Because of significant differences in a large number of baseline clinical and angiographic characteristics between the three groups, propensity score adjustment was performed. Since only pairwise comparisons are allowed in propensity score adjustment, the groups were combined and compared in the following manner: low HDL-C group vs. combined normal-high HDL-C group and combined low-normal HDL-C group vs. high HDL-C group. 


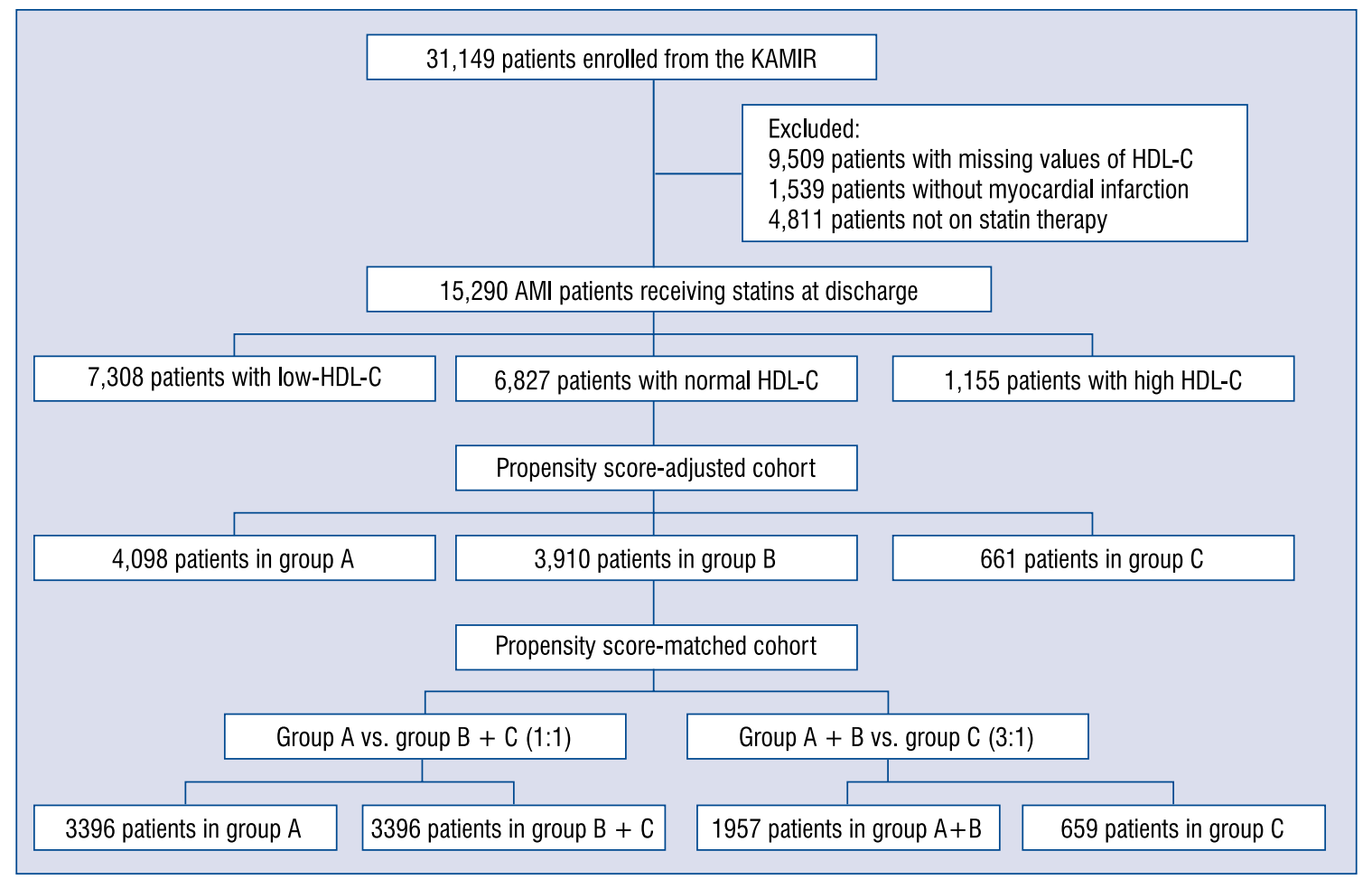

Figure 1. Study flowchart; AMI - acute myocardial infarction; HDL-C — high-density lipoprotein cholesterol; KAMIR — the Korean Acute Myocardial Infarction Registry.

In the main analysis, a propensity score was calculated and used to adjust the between-group comparisons of cardiovascular outcomes [12]. The propensity score was estimated using a nonparsimonious multivariate logistic regression model, with low HDL-C level or high HDL-C level in each subgroup as the dependent variable and characteristics that potentially affect cardiovascular outcomes, including age, sex, history of ischemic heart disease, hypertension, diabetes, dyslipidemia, smoking status, Killip class, number of diseased vessels, post-TIMI flow, initial left ventricular ejection fraction (LVEF), serum creatinine level, serum glucose level, peak troponin I, and use of acetylsalicylic acid, clopidogrel, beta-blockers, angiotensin converting enzyme inhibitors, aldosterone receptor blockers, spironolactone, and insulin.

An additional analysis was performed on matched pairs of patients between each subgroup (group A vs. group $\mathrm{B}+\mathrm{C}$, group $\mathrm{A}+\mathrm{B}$ vs. group $\mathrm{C}$ ). Each propensity score matching was performed using the nearest neighbor method (1:1 for group A vs. group B $+C$, 3:1 for group $A+B$ vs. group $C$ without replacement) with a caliper width of 0.1 . Absolute standardized differences for all covariates before and after matching were estimated to evaluate bias reduction using the propensity score matching method. After propensity score matching, all absolute standardized differences were below $10 \%$, indicating adequate matching. Comparisons of baseline clinical and angiographic characteristics between the matched groups were performed using Student's t-test for continuous variables and the $\chi^{2}$ test for categorical variables.

Risk of negative cardiovascular outcomes between all the subgroups in the propensity scoreadjusted cohort were compared using the Cox proportional hazard regression model with the propensity score as a covariate. Adjusted cumulative survival curves were calculated using the corrected group prognosis method. A secondary analysis was done using the Cox proportional hazard regression model stratified on the matched pairs. Proportional hazard assumptions were tested by using the log-log survival plot and Schoenfeld residuals. Subgroup analysis was also performed only in the propensity score-matched cohort based on the type of MI (STEMI or NSTEMI).

Sensitivity analysis was performed to assess the robustness of results. An inverse probability of treatment weight (IPTW) approach was used because it utilizes the whole patient population rather than the reduced population obtained by the propensity score matching, resulting in greater power and precision [13]. 
A multiple Cox regression analysis was performed to predict risk factors of negative cardiovascular outcome during the follow-up using baseline and angiographic characteristics associated with cardiac death and $\mathrm{MI}$ in a simple Cox regression analysis $(\mathrm{p}<0.1)$ with $>90 \%$ data availability.

All reported $\mathrm{p}$ values were 2 -sided, and $p$ values $<0.05$ were considered to indicate statistically significant differences. All analyses were performed with SPSS 21.0 for Windows (SPSS Inc., Chicago, IL) and $\mathrm{R}$ (version 2.14.2) using freely distributed statistical packages, as well as SAS software, version 9.3 (SAS Institute, Cary, NC, USA).

\section{Results}

Major differences in baseline and angiographic characteristics between the three groups were consistently found in propensity score-adjusted cohort (Table 1). After matching between divided subgroups, all absolute standardized differences were within $10 \%$, which suggests adequate matching.

\section{Overall study outcomes}

In the propensity score-adjusted cohort, 174 $(2 \%)$ patients experienced at least one primary event (cardiovascular death or recurrent MI) during the follow-up period. The rate of primary outcome was highest in low HDL-C group (group A) and similar in normal and high HDL-C groups (groups B and C) (Fig. 2). An increased risk of primary outcome (hazard ratio [HR] 1.755, confidence interval [CI] 1.274-2.417, $\mathrm{p}=0.001$ ) was found in group A, which was driven by an increase in cardiovascular death (HR 2.233, CI 1.465-3.404, $\mathrm{p}<0.001$ ) and eventually in all-cause death (HR 1.486, CI $1.084-2.036, \mathrm{p}=0.014$ ), whereas the risk of primary outcome was not reduced in group C (HR 0.562, CI $0.275-1.146, \mathrm{p}=0.113$ ) (Table 2). There were no differences in the risk of MI recurrence and MACE.

\section{Outcomes in patients with low HDL-C level}

When these analyses were repeated in the matched cohort (group A vs. group B $+\mathrm{C}$ ), risk of primary outcome was increased in Group A (2.5\% vs. $1.5 \%$, HR 1.716 , CI $1.210-2.434, \mathrm{p}=0.002$ ) (Fig. 3A, Table 3). There were no differences in the risks of $\mathrm{MI}$ recurrence, all-cause death, and MACE between the groups.

Analyses by the propensity score matching method were repeated in the subgroups including STEMI and NSTEMI patients. In the matched cohort (group A vs. group B $+\mathrm{C}$ ), low HDL-C level tended to be associated with an increased risk of primary outcome in STEMI patients, but the difference was not statistically significant (HR 1.542 , CI 0.969-2.453, $\mathrm{p}=0.068$ ). In contrast, low HDL-C level was associated with an increased risk of primary outcome in NSTEMI patients (HR 2.058, CI 1.195-3.546, $\mathrm{p}=0.009$ ).

\section{Outcomes in patients with high HDL-C level}

The analysis of the second matched cohort (group A $+\mathrm{B}$ vs. group $\mathrm{C}$ ) revealed a decrease in risk of primary outcome (2.7\% vs. $1.2 \%$, HR 0.449 , CI $0.214-0.946, \mathrm{p}=0.035$ ) (Fig. 3B, Table 3). There were no differences in the risk of secondary outcomes. The analyses by the propensity score matching method were repeated in STEMI and NSTEMI subgroups. Interestingly, high HDL-C level was associated with a reduction in the risk of primary outcome in the STEMI patients (HR 0.267, CI 0.081-0.873, $\mathrm{p}=0.029)$ but not in the NSTEMI patients (HR 0.774, CI 0.292-2.053, $\mathrm{p}=0.606$ ).

\section{Sensitivity analysis}

Sensitivity analyses were performed for the primary outcome using weighted Cox regression by the IPTW method. An increased risk of cardiovascular death and MI was found in the low HDL-C group (HR 1.758, 95\% CI 1.414-2.186, $\mathrm{p}<0.001)$. Furthermore, the risk of cardiovascular death and MI was reduced in the high HDL-C group (HR 0.632, 95\% CI 0.500-0.797, $\mathrm{p}<0.001$ ). This confirmed the results of the main analyses.

\section{Independent predictors \\ of cardiovascular events}

The simple Cox regression analysis of the propensity score-adjusted cohort showed that old age, female sex, high Killip class, history of prior ischemic heart disease, hypertension, diabetes mellitus, three-vessel disease, post-procedural TIMI flow grades 2 and 3, low LVEF, high serum levels of glucose or creatinine at presentation, and high peak serum level of troponin were associated with cardiovascular death and MI during the follow-up (Table 4). After the adjustment for old age, high Killip class, and low LVEF continued to show a significant association with cardiovascular death and MI during the follow-up.

\section{Discussion}

In the present study, the predictive and protective role of HDL-C in AMI patients was inves- 
Table 1. Baseline clinical and procedural characteristics.

\begin{tabular}{|c|c|c|c|c|}
\hline & Group A & Group B & Group C & $\mathbf{P}$ \\
\hline & $\begin{array}{l}\text { Low HDL-C } \\
(n=4,098)\end{array}$ & $\begin{array}{c}\text { Normal HDL-C } \\
(\mathrm{n}=3,910)\end{array}$ & $\begin{array}{l}\text { High HDL-C } \\
(\mathrm{n}=661)\end{array}$ & \\
\hline Age [years] & $62.2 \pm 12.6$ & $63.1 \pm 12.6$ & $64.3 \pm 12.2$ & $<0.001$ \\
\hline Male sex & $5972(78 \%)$ & $7606(70 \%)$ & $590(62.6 \%)$ & $<0.001$ \\
\hline Ischemic heart disease & $1181(15.5 \%)$ & $1525(14.1 \%)$ & $232(14.8 \%)$ & 0.028 \\
\hline Diabetes mellitus & $2418(32.1 \%)$ & $2651(24.7 \%)$ & $317(20.2 \%)$ & $<0.001$ \\
\hline Hypertension & $3804(50.3 \%)$ & $5321(49.5 \%)$ & $753(48.1 \%)$ & 0.251 \\
\hline Dyslipidemia & $956(13.6 \%)$ & $1297(13.0 \%)$ & $157(10.8 \%)$ & 0.014 \\
\hline Smoking & $3735(49.4 \%)$ & $4597(42.7 \%)$ & $578(37.1 \%)$ & $<0.001$ \\
\hline Family history of ischemic heart disease & $659(9.3 \%)$ & $889(8.9 \%)$ & $113(7.8 \%)$ & 0.178 \\
\hline Systolic BP at presentation [mmHg] & $127 \pm 30$ & $130 \pm 27$ & $133 \pm 27$ & $<0.001$ \\
\hline Diastolic BP at presentation [mmHg] & $78 \pm 27$ & $79 \pm 17$ & $81 \pm 16$ & $<0.001$ \\
\hline \multicolumn{5}{|l|}{ Killip class: } \\
\hline 1 & $5412(75.1 \%)$ & $7844(76.3 \%)$ & $1056(70.7 \%)$ & $<0.001$ \\
\hline II & $910(12.6 \%)$ & $1346(13.1 \%)$ & $242(16.2 \%)$ & \\
\hline III & $565(7.8 \%)$ & $752(7.3 \%)$ & $142(9.5 \%)$ & \\
\hline IV & $320(4.4 \%)$ & $336(3.3 \%)$ & $53(3.5 \%)$ & \\
\hline Door-to-balloon time [min] & $173 \pm 196$ & $176 \pm 199$ & $179 \pm 208$ & 0.576 \\
\hline Left ventricular ejection fraction [\%] & $52.4 \pm 11.6$ & $52.5 \pm 13.1$ & $52.2 \pm 15.6$ & 0.538 \\
\hline Peak troponin I [ng/mL] & $39.9 \pm 88.2$ & $44.2 \pm 116.5$ & $49.6 \pm 255.5$ & 0.015 \\
\hline Serum creatinine $[\mathrm{mg} / \mathrm{dL}]$ & $1.25 \pm 1.54$ & $1.10 \pm 1.4$ & $1.05 \pm 1.07$ & $<0.001$ \\
\hline Serum glucose $[\mathrm{mg} / \mathrm{dL}]$ & $171.6 \pm 82.4$ & $165.3 \pm 74.5$ & $164.9 \pm 74.4$ & $<0.001$ \\
\hline Total cholesterol [mg/dL] & $172 \pm 44$ & $189 \pm 42$ & $201 \pm 45$ & $<0.001$ \\
\hline HDL-C [mg/dL] & $33 \pm 5$ & $47 \pm 5$ & $70 \pm 13$ & $<0.001$ \\
\hline LDL-C [mg/dL] & $110 \pm 37$ & $120 \pm 41$ & $117 \pm 42$ & $<0.001$ \\
\hline Triglycerides [mg/dL] & $150 \pm 116$ & $120 \pm 93$ & $102 \pm 88$ & $<0.001$ \\
\hline C-reactive protein [mg/dL] & $11.00 \pm 56.48$ & $9.13 \pm 52.90$ & $8.30 \pm 53.99$ & 0.059 \\
\hline \multicolumn{5}{|l|}{ Medications at discharge: } \\
\hline Acetylsalicylic acid & $7520(98.2 \%)$ & $10661(98.1 \%)$ & $1543(97.7 \%)$ & 0.312 \\
\hline Clopidogrel & $7269(94.9 \%)$ & $10241(94.3 \%)$ & $1455(92.3 \%)$ & $<0.001$ \\
\hline Calcium channel blockers & $734(9.8 \%)$ & $1075(10.1 \%)$ & $171(11.1 \%)$ & 0.3 \\
\hline Beta-adrenergic blockers & $6061(79.5 \%)$ & $8534(78.8 \%)$ & $1217(77.4 \%)$ & 0.148 \\
\hline ACE inhibitors & $4710(62 \%)$ & $6972(64.8 \%)$ & $1032(65.9 \%)$ & $<0.001$ \\
\hline Angiotensin receptor blockers & $1652(22 \%)$ & $2228(20.8 \%)$ & $291(18.8 \%)$ & 0.011 \\
\hline Spironolactone & $621(8.3 \%)$ & $901(8.5 \%)$ & $120(7.8 \%)$ & 0.645 \\
\hline Insulin & $127(1.7 \%)$ & $152(1.4 \%)$ & $12(0.8 \%)$ & 0.02 \\
\hline Oral hypoglycemic agents & $1201(15.7 \%)$ & $1499(13.8 \%)$ & $214(13.5 \%)$ & 0.001 \\
\hline Number of diseased vessels: & & & & $<0.001$ \\
\hline 1 & $2861(40.4 \%)$ & $4588(46 \%)$ & $701(51.4 \%)$ & \\
\hline 2 & $2180(30.8 \%)$ & $2966(29.7 \%)$ & $402(29.5 \%)$ & \\
\hline 3 & $1808(25.5 \%)$ & $2140(21.4 \%)$ & $232(17 \%)$ & \\
\hline Left main disease & $229(3.2 \%)$ & $288(2.9 \%)$ & $30(2.2 \%)$ & \\
\hline Culprit lesion: & & & & $<0.001$ \\
\hline Left anterior descending & $3022(42.9 \%)$ & $4952(49.8 \%)$ & $747(54.9 \%)$ & \\
\hline Left circumflex & $1238(17.6 \%)$ & $1712(17.2 \%)$ & $228(16.8 \%)$ & \\
\hline Right coronary artery & $2646(37.6 \%)$ & $3111(31.3 \%)$ & $360(26.5 \%)$ & \\
\hline Left main tract & $134(1.9 \%)$ & $170(1.7 \%)$ & $26(1.9 \%)$ & \\
\hline Successful PCl & $6466(97.6 \%)$ & $9161(97.5 \%)$ & $1226(96.8 \%)$ & 0.403 \\
\hline Post-procedural TIMI flow grade: & & & & 0.125 \\
\hline 0 & $112(1.7 \%)$ & $116(1.3 \%)$ & $23(1.8 \%)$ & \\
\hline 1 & $61(0.9 \%)$ & $79(0.9 \%)$ & $13(1 \%)$ & \\
\hline 2 & $229(3.6 \%)$ & $354(3.9 \%)$ & $56(4.5 \%)$ & \\
\hline 3 & $6023(93.7 \%)$ & $8542(94 \%)$ & $1155(92.6 \%)$ & \\
\hline
\end{tabular}

ACE - angiotensin converting enzyme; BP — blood pressure; HDL-C — high-density lipoprotein cholesterol; LDL-C — low-density lipoprotein cholesterol; $\mathrm{PCl}$ - percutaneous coronary intervention; TIMI — thrombolysis in myocardial infarction 


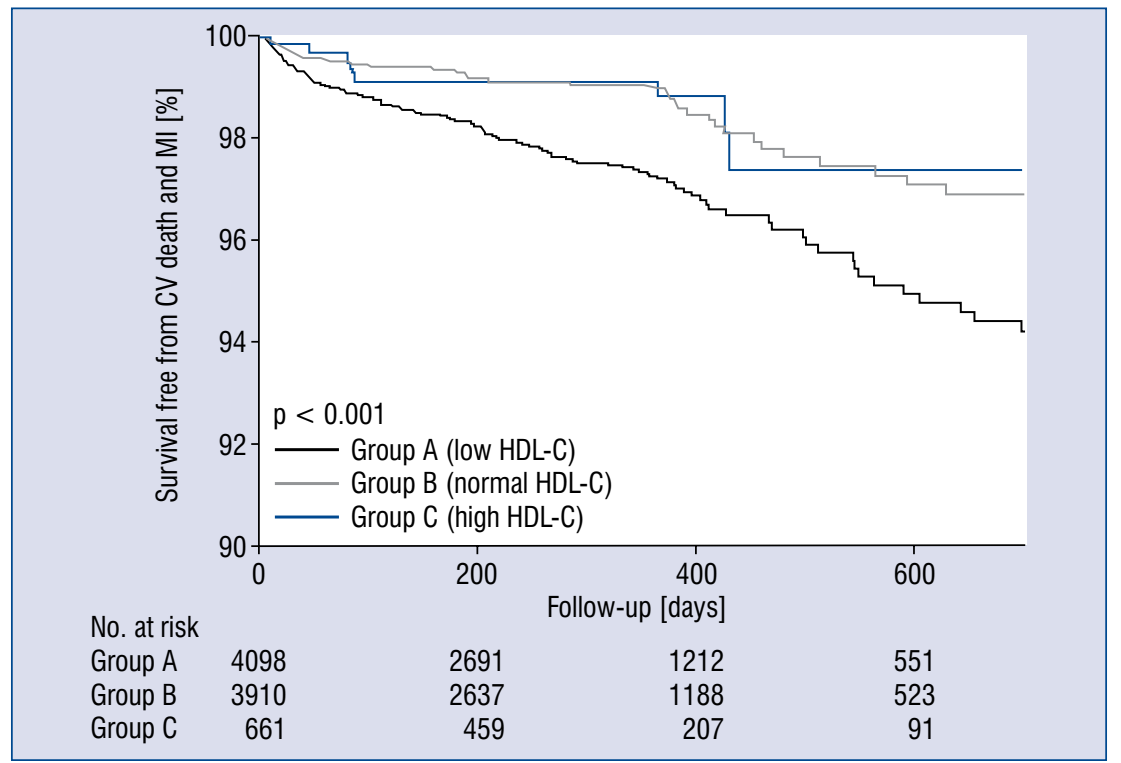

Figure 2. Survival curves free from cardiovascular (CV) death and myocardial infarction (MI) during follow-up in the propensity score-adjusted cohort; HDL-C — high-density lipoprotein cholesterol.

Table 2. The risk of negative clinical outcomes for patients with low and high high-density lipoprotein cholesterol (HDL-C) level in the propensity-adjusted cohort.

\begin{tabular}{|c|c|c|c|c|}
\hline & \multicolumn{2}{|c|}{ Low HDL-C } & \multicolumn{2}{|c|}{ High HDL-C } \\
\hline & HR $(95 \% \mathrm{Cl})$ & $\mathbf{P}$ & HR (95\% Cl) & $\mathbf{P}$ \\
\hline Cardiovascular death and MI & $1.755(1.274-2.417)$ & 0.001 & $0.562(0.275-1.146)$ & 0.113 \\
\hline Cardiovascular death & $2.233(1.465-3.404)$ & $<0.001$ & $0.775(0.359-1.675)$ & 0.517 \\
\hline Recurrent MI & $1.203(0.725-1.996)$ & 0.474 & $0.192(0.027-1.389)$ & 0.102 \\
\hline All-cause death & $1.486(1.084-2.036)$ & 0.014 & $0.964(0.556-1.673)$ & 0.897 \\
\hline MACE & $1.022(0.877-1.190)$ & 0.65 & $0.915(0.697-1.202)$ & 0.524 \\
\hline
\end{tabular}

$\mathrm{Cl}$ — confidence interval; $\mathrm{HR}$ — hazard ratio; $\mathrm{MI}$ - myocardial infarction; MACE — major adverse cardiac event

tigated. evidence was found that the initial level of HDL-C affected cardiovascular outcomes in a cohort of Asian patients with acute AMI that were treated with statins. Thus, the rate of cardiovascular death and MI recurrence was higher in the patients with low HDL-C level, whereas high HDL-C level was associated with a lower rate of major cardiovascular events. Importantly, the application of different adjustment methods did not affect these findings. Moreover, subgroup analyses revealed that low HDL-C level was associated with a significantly higher risk of major cardiovascular events in the NSTEMI patients and only a modestly increased risk in the STEMI patients. Finally, high HDL-C level was associated with a lower risk of major cardiovascular events only in STEMI patients.
Lower HDL-C levels have been shown to be associated with a higher risk of cardiovascular events and greater severity of atherosclerosis even in patients with lower LDL levels, including those treated with statins [2]. Moreover, several studies have demonstrated that low HDL-C level in NSTEMI patients was predictive of major adverse cardiovascular events $[5,14]$. In addition, low initial HDL-C level was associated with a significantly higher risk of cardiovascular events in STEMI patients [15]. However, the design of the above studies included the use of a constant value of HDL-C, and baseline and angiographic characteristics that might affect clinical outcomes were not properly compensated for. In order to rule out confounding effects of covariates, a propensity score model was built and two 1:1 cohorts of 


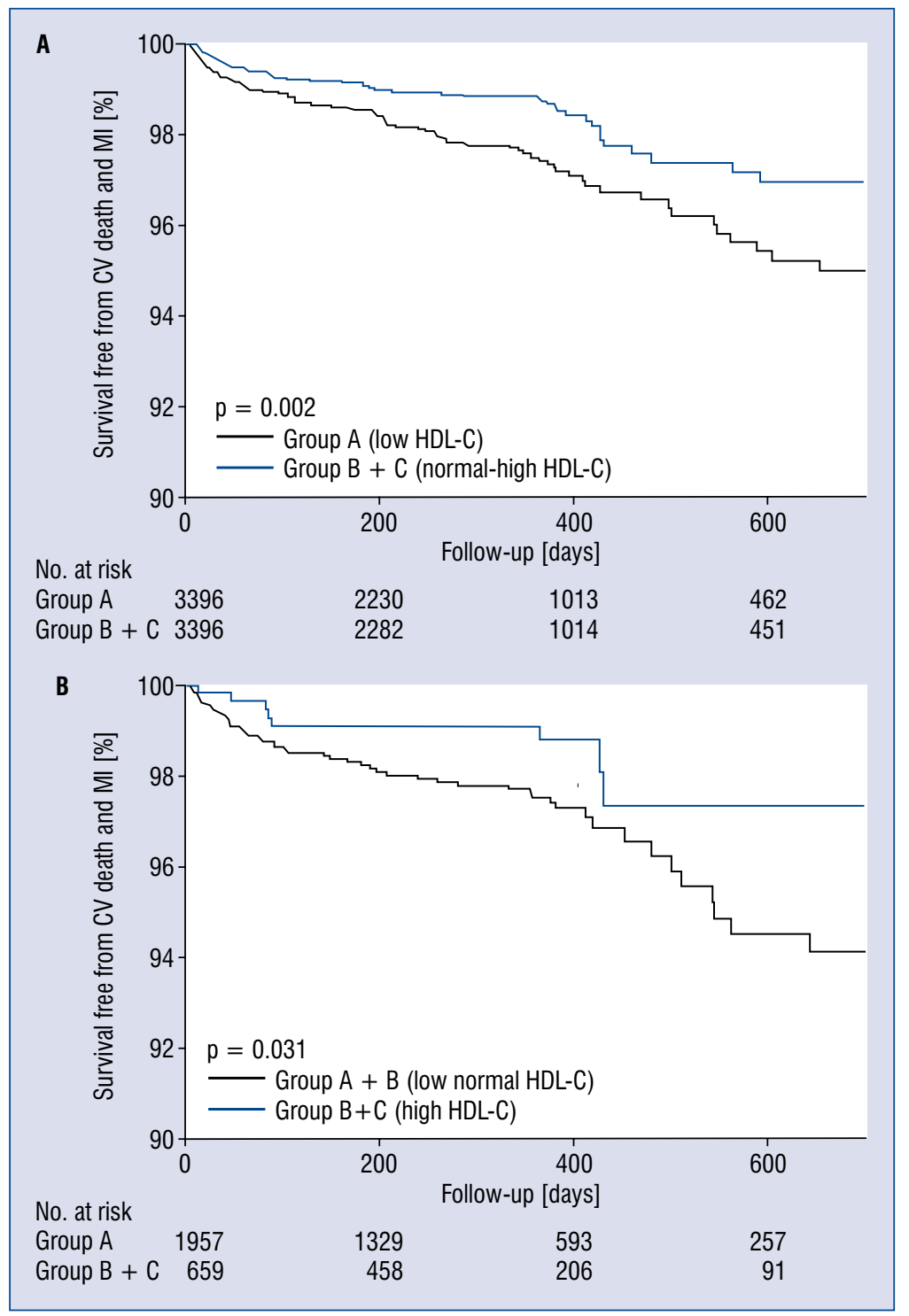

Figure 3. Survival curves free from cardiovascular death and myocardial infarction during follow-up in Group A vs. Group B + C (A) and Group A+B vs. Group C (B) in the propensity score-matched cohort; CV — cardiovascular; HDL-C — high-density lipoprotein cholesterol; $\mathrm{MI}$ - myocardial infarction.

optimally matched patients were obtained in the present study. Low initial HDL-C level was found to directly correlate with cardiovascular events (HR 1.716). Moreover, a consistent result (HR 1.758) was obtained using IPTW methods. The present analysis strengthens the notion that low HDL-C levels may be a predictor of cardiovascular outcomes in AMI patients.

The vasoprotective effect of HDL-C is thought to be related to reverse macrophage cholesterol transport [16]. Furthermore, it has been recently found that HDL-C facilitates endothelial homeostasis via the increase in nitric oxide production as well as the inhibition of critical pathways involved in vascular inflammation and endothelial apoptosis. It has also been reported that the ability of HDL-C to stimulate nitrate oxide production and promote endothelial repair is impaired in patients with CAD [17]. Based on these observations, the term "dysfunctional HDL-C" was introduced, which indicates the loss of anti-inflammatory and vasoprotective effects [8]. The results of a recent study that used the values of $>50 \mathrm{mg} / \mathrm{dL}$ for women and $>40 \mathrm{mg} / \mathrm{dL}$ for men, as a definition of high HDL-C level have suggested that higher HDL-C levels are not associated with reduced risk of vascular events in CAD patients [18]. In the present study, high HDL-C level as $>60 \mathrm{mg} / \mathrm{dL}$ were defined accord- 
Table 3. The incidence and risk of negative clinical outcomes in patients with low and high high-density lipoprotein cholesterol (LDL-C and HDL-C) level in the propensity-matched cohort.

\begin{tabular}{|c|c|c|c|c|}
\hline Low HDL-C & $\begin{array}{c}\text { Group A } \\
(n=3,396)\end{array}$ & $\begin{array}{c}\text { Group B + C } \\
(\mathrm{n}=3,396)\end{array}$ & HR (95\% Cl) & $\mathbf{P}$ \\
\hline Cardiovascular death and MI & $85(2.5 \%)$ & $50(1.5 \%)$ & $1.716(1.210-2.434)$ & 0.002 \\
\hline Cardiovascular death & $57(1.7 \%)$ & $29(0.9 \%)$ & $1.981(1.267-3.099)$ & 0.003 \\
\hline Recurrent MI & $28(0.8 \%)$ & $21(0.6 \%)$ & $1.348(0.765-2.377)$ & 0.301 \\
\hline All-cause death & $78(2.3 \%)$ & $58(1.7 \%)$ & $1.365(0.972-1.918)$ & 0.073 \\
\hline MACE & $287(8.5 \%)$ & $284(8.4 \%)$ & $1.007(0.855-1.187)$ & 0.931 \\
\hline High HDL-C & $\begin{array}{c}\text { Group } A+B \\
(n=1,957)\end{array}$ & $\begin{array}{l}\text { Group C } \\
(\mathrm{n}=651)\end{array}$ & HR (95\% CI) & $\mathbf{P}$ \\
\hline Cardiovascular death and MI & $52(2.7 \%)$ & $8(1.2 \%)$ & $0.449(0.214-0.946)$ & 0.035 \\
\hline Cardiovascular death & $34(1.7 \%)$ & $7(1.1 \%)$ & $0.604(0.268-1.363)$ & 0.225 \\
\hline Recurrent MI & $18(0.9 \%)$ & $1(0.2 \%)$ & $0.161(0.021-1.203)$ & 0.075 \\
\hline All-cause death & $50(2.6 \%)$ & $14(2.1 \%)$ & $0.824(0.456-1.491)$ & 0.523 \\
\hline MACE & $162(8.3 \%)$ & $57(8.6 \%)$ & $1.028(0.760-1.391)$ & 0.856 \\
\hline
\end{tabular}

$\mathrm{Cl}$ - confidence interval; $\mathrm{HR}$ - hazard ratio; $\mathrm{Ml}$ - myocardial infarction; MACE — major adverse cardiac event

Table 4. Independent predictors of cardiovascular death and myocardial infarction.

\begin{tabular}{|c|c|c|c|c|}
\hline \multirow[t]{2}{*}{ Variable } & \multicolumn{2}{|c|}{ Simple Cox regression } & \multicolumn{2}{|c|}{ Multiple Cox regression } \\
\hline & HR (95\% CI) & $\mathbf{P}$ & HR $(95 \% \mathrm{CI})$ & $\mathbf{P}$ \\
\hline Age (1-year increase) & $1.042(1.029-1.056)$ & $<0.001$ & $1.035(1.020-1.051)$ & $<0.001$ \\
\hline Female sex & $1.716(1.265-2.327)$ & $<0.001$ & & \\
\hline Killip class $3 / 4$ & $3.281(2.329-4.621)$ & $<0.001$ & $1.616(1.106-2.359)$ & 0.001 \\
\hline Ischemic heart disease & $1.588(1.088-2.318)$ & 0.016 & & \\
\hline Hypertension & $1.411(1.046-1.903)$ & 0.024 & & \\
\hline Diabetes mellitus & $1.570(1.150-2.143)$ & 0.004 & & \\
\hline Dyslipidemia & $1.058(0.692-1.618)$ & 0.793 & & \\
\hline Current smoking & $0.768(0.566-1.042)$ & 0.089 & & \\
\hline Post-procedural TIMI flow grade $2-3$ & $0.766(0.284-2.066)$ & 0.598 & & \\
\hline Three-vessel disease & $1.980(1.458-2.688)$ & $<0.001$ & & \\
\hline Low LVEF & $0.947(0.936-0.959)$ & $<0.001$ & $0.962(0.948-0.975)$ & $<0.001$ \\
\hline Serum glucose level & $1.002(1.001-1.004))$ & 0.002 & & \\
\hline Serum creatinine level & 1.130 (1.048-1.219) & 0.001 & & \\
\hline Serum peak troponin level & $1.001(1.000-1.001)$ & 0.043 & & \\
\hline
\end{tabular}

$\mathrm{Cl}$ - confidence interval; HR - hazard ratio; LVEF — left ventricular ejection fraction; TIMI — thrombolysis in myocardial infarction

ing to ATP III guidelines. According to results of the propensity-based analysis, high HDL-C level was associated with reduced risk of cardiovascular events (HR 0.449) in AMI patients. This finding was confirmed by the use of IPTW methods (HR 0.632). Therefore, this analysis suggests that high initial HDL-C level might protect against cardiovascular events in AMI patients.
Several clinical trials and meta-analyses indicated that raising HDL-C might reduce future cardiovascular events and also the atherosclerotic burden itself. Several studies which were conducted based on the modulation of HDL-C by agonizing nuclear transcription factors and the enzyme responsible for HDL metabolism in serum have failed to demonstrate the protection against future cardiac 
events [19]. HDL metabolism and modulation were far more complex than was thought, and changes in specific circumstances such as disease entity and disease progression [20]. Focus now moved to modulating various sub-fraction of HDL-C, which might lead to reduced residual risk after statin therapy [21].

\section{Limitations of the study}

The main limitation of the present study is its retrospective nature. Despite the use of propensity score analysis and sensitivity analysis that employed the IPTW approach to control for selection bias, unidentified confounders may have influenced the results. Furthermore, several studies have suggested that serum lipid levels after AMI may not represent true baseline levels [22], which may potentially have affected results. However, other reports indicate that HDL-C levels obtained within the first 24 to 48 hours subsequent tohospital admission are a reliable measure of true baseline status [23, 24], which justifiesthis approach to the HDL-C level measurements. Finally, lipid profile was not monitored during the follow-up period.

\section{Conclusions}

Initial HDL-C levels may predict cardiovascular events in AMI patients undergoing intensive statin treatment. In particular, low HDL-C levels were associated with increased incidence of cardiovascular death and recurrent MI. Furthermore, high HDL-C levels were possibly associated with reduced risk of cardiovascular events, especially in STEMI patients, indicating a potential protective effect.

Funding: This work was supported by a clinical research grant from Pusan National University Hospital (2015).

\section{Conflict of interest: None declared}

\section{References}

1. Jafri H, Alsheikh-Ali AA, Karas RH. Meta-analysis: statin therapy does not alter the association between low levels of highdensity lipoprotein cholesterol and increased cardiovascular risk. Ann Intern Med. 2010; 153(12): 800-808, doi: 10.7326/00034819-153-12-201012210-00006, indexed in Pubmed: 21173414.

2. Barter P, Gotto AM, LaRosa JC, et al. Treating to New Targets Investigators. HDL cholesterol, very low levels of LDL cholesterol, and cardiovascular events. N Engl J Med. 2007; 357(13): 1301-1310, doi: 10.1056/NEJMoa064278, indexed in Pubmed: 17898099.
3. Olsson AG, Schwartz GG, Szarek M, et al. High-density lipoprotein, but not low-density lipoprotein cholesterol levels influence short-term prognosis after acute coronary syndrome: results from the MIRACL trial. Eur Heart J. 2005; 26(9): 890-896, doi: 10.1093/eurheartj/ehi186, indexed in Pubmed: 15764620.

4. Duffy D, Rader DJ. Update on strategies to increase HDL quantity and function. Nat Rev Cardiol. 2009; 6(7): 455-463, doi: 10.1038/nrcardio.2009.94, indexed in Pubmed: 19488077.

5. Acharjee S, Roe MT, Amsterdam EA, et al. Relation of admission high-density lipoprotein cholesterol level and in-hospital mortality in patients with acute non-ST segment elevation myocardial infarction (from the National Cardiovascular Data Registry). Am J Cardiol. 2013; 112(8): 1057-1062, doi: 10.1016/j. amjcard.2013.05.050, indexed in Pubmed: 23891245.

6. Fabregat-Andrés Ó, Ferrando-Beltrán M, Lucas-Inarejos E, et al. High-density lipoproteins and myocardial necrosis in patients with acute myocardial infarction and ST segment elevation. Rev Clin Esp (Barc). 2013; 213(2): 75-80, doi: 10.1016/j. rce.2012.07.008, indexed in Pubmed: 23182648.

7. Besler C, Heinrich K, Rohrer L, et al. Mechanisms underlying adverse effects of HDL on eNOS-activating pathways in patients with coronary artery disease. J Clin Invest. 2011; 121(7): 2693-2708, doi: 10.1172/JCI42946, indexed in Pubmed: 21701070.

8. Besler C, Lüscher TF, Landmesser U. Molecular mechanisms of vascular effects of High-density lipoprotein: alterations in cardiovascular disease. EMBO Mol Med. 2012; 4(4): 251-268, doi: 10.1002/emmm.201200224, indexed in Pubmed: 22431312.

9. Jeong YAh, Jeong MHo, Jeong HC, et al. Korea Acute Myocardial Infarction Registry (KAMIR) Investigators. Impact of smoking on clinical outcomes in female patients with acute myocardial infarction. Korean Circ J. 2015; 45(1): 22-27, doi: 10.4070/ kcj.2015.45.1.22, indexed in Pubmed: 25653700.

10. Sim DS, Jeong MHo, Cho KH, et al. Other Korea Acute Myocardial Infarction Registry (KAMIR) Investigators. Effect of early statin treatment in patients with cardiogenic shock complicating acute myocardial infarction. Korean Circ J. 2013; 43(2): 100-109, doi: 10.4070/kcj.2013.43.2.100, indexed in Pubmed: 23508129.

11. National Cholesterol Education Program (NCEP) Expert Panel. Third Report of the National Cholesterol Education Program (NCEP) Expert Panel on Detection, Evaluation and Treatment of High Blood Cholesterol in Adults (Adult Treatment Panel III). Arch Intern Med. 2002; 6: 284.

12. Robins JM, Hernán MA, Brumback B. Marginal structural models and causal inference in epidemiology. Epidemiology. 2000; 11(5): 550-560, indexed in Pubmed: 10955408.

13. Xie J, Liu C. Adjusted Kaplan-Meier estimator and log-rank test with inverse probability of treatment weighting for survival data. Stat Med. 2005; 24(20): 3089-3110, doi: 10.1002/sim.2174, indexed in Pubmed: 16189810.

14. Wolfram RM, Brewer HB, Xue Z, et al. Impact of low highdensity lipoproteins on in-hospital events and one-year clinical outcomes in patients with non-ST-elevation myocardial infarction acute coronary syndrome treated with drug-eluting stent implantation. Am J Cardiol. 2006; 98(6): 711-717, doi: 10.1016/j. amjcard.2006.04.006, indexed in Pubmed: 16950168.

15. Ji MiS, Jeong MHo, Ahn YK, et al. Korea Acute Myocardial Infarction Registry Investigators. Impact of low level of high-density lipoprotein-cholesterol sampled in overnight fasting state on the clinical outcomes in patients with acute myocardial infarction (difference between ST-segment and non-ST-segmentelevation myocardial infarction). J Cardiol. 2015; 65(1): 63-70, doi: 10.1016/j.jjcc.2014.04.002, indexed in Pubmed: 25242301. 
16. Khera AV, Cuchel M, de la Llera-Moya M, et al. Cholesterol efflux capacity, high-density lipoprotein function, and atherosclerosis. N Engl J Med. 2011; 364(2): 127-135, doi: 10.1056/ NEJMoa1001689, indexed in Pubmed: 21226578.

17. Sorrentino SA, Besler C, Rohrer L, et al. Endothelial-vasoprotective effects of high-density lipoprotein are impaired in patients with type 2 diabetes mellitus but are improved after extended-release niacin therapy. Circulation. 2010; 121(1): 110-122, doi: 10.1161/ CIRCULATIONAHA.108.836346, indexed in Pubmed: 20026785.

18. Angeloni E, Paneni F, Landmesser U, et al. Lack of protective role of HDL-C in patients with coronary artery disease undergoing elective coronary artery bypass grafting. Eur Heart J. 2013; 34(46): 3557-3562, doi: 10.1093/eurheartj/eht163, indexed in Pubmed: 23704708.

19. Toth PP, Barylski M, Nikolic D, et al. Should low high-density lipoprotein cholesterol (HDL-C) be treated? Best Pract Res Clin Endocrinol Metab. 2014; 28(3): 353-368, doi: 10.1016/j. beem.2013.11.002, indexed in Pubmed: 24840264.

20. Barylski M, Toth PP, Nikolic D, et al. Emerging therapies for raising high-density lipoprotein cholesterol (HDL-C) and augment- ing HDL particle functionality. Best Pract Res Clin Endocrinol Metab. 2014; 28(3): 453-461, doi: 10.1016/j.beem.2013.11.001, indexed in Pubmed: 24840270.

21. Garcia-Rios A, Nikolic D, Perez-Martinez P, et al. LDL and HDL subfractions, dysfunctional HDL: treatment options. Curr Pharm Des. 2014; 20(40): 6249-6255, doi: 10.2174/13816128206661406 20154014, indexed in Pubmed: 24953394.

22. Ryder RE, Hayes TM, Mulligan IP, et al. How soon after myocardial infarction should plasma lipid values be assessed? $\mathrm{Br}$ Med J (Clin Res Ed). 1984; 289(6459): 1651-1653, doi: 10.1136/ bmj.289.6459.1651, indexed in Pubmed: 6439361.

23. Pitt B, Loscalzo J, Ycas J, et al. Lipid levels after acute coronary syndromes. J Am Coll Cardiol. 2008; 51(15): 1440-1445, doi: 10.1016/j.jacc.2007.11.075, indexed in Pubmed: 18402897.

24. Barth JH, Jackson BM, Farrin AJ, et al. SPACE ROCKET Trial Group. Change in serum lipids after acute coronary syndromes: secondary analysis of SPACE ROCKET study data and a comparative literature review. Clin Chem. 2010; 56(10): 1592-1598, doi: 10.1373/clinchem.2010.145631, indexed in Pubmed: 20729301. 